\title{
Clinicopathological characteristics, treatment, and survival of pulmonary large cell neuroendocrine carcinoma: A SEER Population-Based Study
}

\author{
Ling Cao ${ }^{1}$, Zhi-wen Li ${ }^{2}$, Min Wang ${ }^{3}$, Ting-ting Zhang ${ }^{4}$ ， Bo Bao ${ }^{1}$, Yun-peng Liu ${ }^{\text {Corresp. } 5}$ \\ 1 Department of Radiation Oncology, Cancer Hospital of Jilin Province, Changchun, Jilin, China \\ 2 Department of Anesthesiology, First Hospital of Jilin University, Changchun, Jilin, China \\ 3 Department of Pathology, Cancer Hospital of Jilin Province, Changchun, China \\ 4 Department of Medical Oncology, Cancer Hospital of Jilin Province, Changchun, China \\ 5 Department of Thoracic Surgery, First Hospital of Jilin University, Changchun, Jilin, China \\ Corresponding Author: Yun-peng Liu \\ Email address: liuyunpeng1848@163.com
}

Background: This study was designed to investigate the clinicopathological characteristics, treatment and survival of patients with pulmonary large cell neuroendocrine carcinoma (LCNEC).

Methods: The Surveillance, Epidemiology and End Results (SEER) database was utilized to identify patients diagnosed with pulmonary LCNEC between 2004 and 2013. Kaplan-Meier analysis was conducted to determine the overall survival (OS) and cancer-specific survival (CSS) rate. Univariate survival analysis along with log-rank test, and Cox proportional hazards model were employed to detect independent prognostic factors.

Results: Pulmonary LCNEC accounted for $0.58 \%$ 2972/510607) of the total number of lung and bronchus carcinoma. And a total of 1530 eligible cases were identified, with the median follow-up time of 11 months. To be specific, the 3-, 5-year OS and CSS rates were $22.8 \%, 16.8 \%$ and $26.5 \%, 20.8 \%$ respectively. Generally, pulmonary LCNEC was commonly detected in the elderly (72.2\%), males (55.9\%), the upper lobe $(62.0 \%)$ and advanced AJCC stage (65.5\%). Multivariate analysis revealed that elderly [ $\geq 60$ and $<80$ years) HR:1.203, 95\%Cl: $1.053-1.375, \mathrm{P}=0.007 ;(\geq 80$ years)HR: $1.530,95 \% \mathrm{Cl}: 1.238-1.891, \mathrm{P}<0.001$ ], and advanced AJCC stage [(stage III)HR:2.606, 95\%Cl: 2.083-3.260,P<0.001; (stage IV)HR:4.881, 95\%Cl: 3.923-6.072,P<0.001] were independent unfavorable prognostic factors, and that female (HR: $0.845,95 \% \mathrm{Cl}: 0.754-0.947, \mathrm{P}=0.004)$ ), surgery [(Segmentectomy/wedge resection) HR:0.526, 95\%Cl: 0.413-0.669,P<0.001;(Lobectomy / Bilobectomy) HR:0.357, 95\%Cl: 0.290-0.440, P<0.001;(Pneumonectomy) HR:0.491, 95\%Cl: $0.355-0.679, \mathrm{P}<0.001$ ] , chemotherapy (HR:0.442, 95\% $\mathrm{Cl}: 0.389-0.503, \mathrm{P}<0.001)$, and radiation (HR:0.837, 95\% Cl: $0.738-0.949, \mathrm{P}=0.005$ ) were independent favorable prognostic factors. 
Conclusion: To sum up, age at diagnosis, sex, AJCC 8th edition stage, surgery, chemotherapy and radiation were significantly associated with OS of patients with pulmonary LCNEC. 
1 Clinicopathological characteristics, treatment, and survivalof pulmonary 2 large cell neuroendocrine carcinoma: A SEER Population-Based Study

3 Ling $\mathrm{Cao}^{1 *}$,Zhi-wen $\mathrm{Li}^{{ }^{2 *}}$,Min Wang ${ }^{3}$, Ting-ting $\mathrm{Zhang}^{4}$, Bo bao ${ }^{1}$, Y un-peng Liu ${ }^{5}$

$4{ }^{1}$ Department of Radiation Oncology, Cancer Hospital of Jilin Province, NO.1018 Huguang Road 5 , Changchun ,130012,Jilin,China.

$6{ }^{2}$ Department of Anesthesiology, First Hospital of Jilin University, Changchun, Jilin,130021,

7 China.

8 3epartment of Pathology, Cancer Hospital of Jilin Province, NO.1018 HuguangRoad, 9 Changchun, 130012,Jilin, China.

$10{ }^{4}$ Department of Medical Oncology, Cancer Hospital of Jilin Province, NO.1018 Huguang Road, 11 Changchun ,130012,Jilin, China.

$12{ }^{5}$ Department of Thoracic Surgery, First Hospital of Jilin University, Changchun, Jilin,130021,

13 China.

14 China. Tel: +86-431-85871227; Fax: +86-431-85879125

\section{Corresponding author:}

Yun-peng Liu, MD. (liuyunpeng1848@163.com).Mailing address: Department of Thoracic Surgery, The First Hospital of Jilin University, No. 71 Xinmin Street, Changchun, 130012, 


\section{Abstract}

Background: This study was designed to investigate the clinicopathological characteristics, treatment and survival of patients with pulmonary large cell neuroendocrine carcinoma (LCNEC).

Methods: The Surveillance, Epidemiology and End Results (SEER) database was utilized to identify patients diagnosed with pulmonary LCNEC between 2004 and 2013. Kaplan-Meier analysis was conducted to determine the overall survival (OS) and cancer-specific survival (CSS) rate. Univariate survival analysis along with log-rank test, and Cox proportional hazards model were employed to detect independent prognostic factors.

Results: Pulmonary LCNEC accounted for 0.58\% (2972/510607) of the total number of lung and bronchus carcinoma. And a total of 1530 eligible cases were identified, with the median follow-up time of 11 months. To be specific, the 3-, 5-year OS and CSS rates were 22.8\%, 16.8\% and $26.5 \%, 20.8 \%$ respectively. Generally, pulmonary LCNEC was commonly detected in the elderly (72.2\%), males (55.9\%), the upper lobe (62.0\%) and advanced AJCC stage (65.5\%). Multivariate analysis revealed that elderly $[(\geq 60$ and $<80$ years $)$ HR:1.203, 95\%CI: 1.053$1.375, P=0.007 ;(\geq 80$ years $) \mathrm{HR}: 1.530,95 \% \mathrm{CI}: 1.238-1.891, P<0.001]$, and advanced AJCC stage [(stage III)HR:2.606, 95\%CI: 2.083-3.260,P<0.001; (stage IV)HR:4.881, 95\%CI: $3.923-$ 6.072,P<0.001] were independent unfavorable prognostic factors, and that female (HR: 0.845 , 95\%CI: 0.754-0.947,P=0.004)), surgery [(Segmentectomy/wedge resection) HR:0.526, 95\%CI: 
52

53

54

55

56

57

58

59

60

61

62

63

64

65

66

67

68

69

70

71

72

0.413-0.669,P<0.001;(Lobectomy ／ Bilobectomy) HR:0.357, 95\%CI: 0.290-0.440, $P<0.001$;(Pneumonectomy) HR:0.491, 95\%CI: 0.355-0.679,P<0.001], chemotherapy (HR:0.442, 95\%CI: $0.389-0.503, P<0.001)$, and radiation (HR:0.837, 95\%CI: $0.738-0.949, P=0.005)$ were independent favorable prognostic factors.

Conclusion: To sum up, age at diagnosis, sex, AJCC 8th edition stage, surgery, chemotherapy and radiation were significantly associated with OS of patients with pulmonary LCNEC.

(1)

(1)

(19)

2

3 


\section{Introduction}

Pulmonary large cell neuroendocrine carcinoma (LCNEC) is an extremely rare disease with an incidence accounting for approximately $3 \%$ of all types of lung cancers [1]. LCNEC was first

suggested by Travis et al. in 1991 as a new type of solitary pulmonary neuroendocrine tumor, which was different from the typical, atypical carcinoid or small cell lung carcinoma (SCLC) [2]. In the following 1999 and 2004, the World Health Organization (WHO) admitted that LCNEC was a variant of large cell carcinoma (LCC), belonging to neuroendocrine tumors and one of the non-small cell lung cancer[3, 4].

Due to its low incidence and a lack of relevant clinical trial data, there is limited clinical understanding of its biological characteristics; hence, there are some controversies over the therapeutic strategies, especially in advanced patients.[1]. Generally, most clinical studies of LCNEC are limited to retrospective studies using sample sizes less than 100 cases; the largest sample size published only included 127 cases [5]. In 2018, there was also a retrospective study concerning the prognosis of LCNEC by enrolling 126 patients [6]. However, these studies were all of small samples, with greatly varied results, therefore, it is necessary to conduct large-scale, multi-center clinical studies.

The Surveillance, Epidemiology and End Results (SEER) program is supported by the National Cancer Institute (NCI).This program contains research data of 18 different populationbased cancer registries, which covers $30 \%$ of the United States population [7]. Hence, a number of studies on rare diseases have been performing by using large samples from the SEER database 
[8-10]. However, studies on pulmonary LCNEC from SEER database have not been found. In consideration of a lack of the published data on pulmonary LCNEC and the needs from clinical work, we performed an analysis of the SEER database to characteristics, prognosis and survival of patients with this disease.

\section{Materials and Methods}

\section{Ethics statement}

The SEER Research Data Agreement was signed for accessing SEER information with the use of reference number 16462-Nov2016. Following approved guidelines, we performed the research methods to obtain data provided from the SEER database. The data analysis was considered by the Office for Human Research Protection to be non-human subjects who were researched by the United States Department of Health and Human Services, as they were publicly available and de-identified. Thus, it did not require any approval by the institutional review board.

\section{Study population}

Patient data were obtained using the SEER database (Submission, November 2016). The SEER*State v8.3.5 tool, released on March 6, 2018, was used for determining and selecting eligible patients. Additionally, the study duration ranged from January 1, 2004 to December 31, 2013(because details about tumor size and extension were not available in the SEER database before 2004). The inclusion criteria were listed as follows: age at the diagnosis $\geq 20$ years;

LCNEC pathologically confirmed based on histology (ICD-O-3 8013/3); restriction on site 
recodes ICD-O-3/WHO 2008 (International Classification of Diseases for Oncology, Third Edition) to "Lung and Bronchus". The exclusion criteria were as follows: (1) patients younger than20 years old; (2) patients with more than one primary cancer; (3) patients with missing or incomplete survival data; (4) without pathological confirmation based on histology;(4)patients with low grade pathology (Grade I and Grade II) were excluded because LCNEC was a kind of high grade neuroendocrine lung tumors; (5) patients without certain important clinicopathological information, including age, race, marital status, primary site, surgical type and AJCC stage. The remaining patients were defined as SEER primary cohort.

Covariates

Covariates included the age at diagnosis ( $<60$ years; $\geq 60$ and $<80$ years; $\geq 80$ years), gender(male; female), race/ethnicity (white; black; other), marital status(married; unmarried),primary site (main bronchus; upper lobe; middle lobe; main bronchus; lower lobe; overlapping lesion of lung), laterality(bilateral; left; right), differentiation (poorly differentiated, Grade III; undifferentiated, Grade IV; unknown), AJCC stage groups (8th edition) (I; II; III; IV), surgery (no surgery; segmentectomy/wedge resection; lobectomy / bilobectomy; pneumonectomy), chemotherapy(no/unknown; yes),radiation(no/unknown; yes).Overlapping was defined as cancer extents over more than one lobe. The widowed or single (never married or having a domestic partner) or divorced or separated patients were classified as unmarried. 8th edition stage group was then calculated for each patient according to tumor size, extension and 7th edition N/M stages.

The endpoints of this study were overall survival (OS), which was defined as the time 
interval from diagnosis to the most recent follow-up date, or date of death. Cancer-specific survival (CSS) was defined as the time interval from diagnosis to the most recent follow-up date or date of death caused by pulmonary LCNEC. There was a predetermined cut-off date based on the SEER 2016 submission database, containing death information until 2014. Therefore, the cut-off date was set at December 31, 2014 in this study.

\section{Statistical analysis}

The Kaplan-Meier (KM) method was used to estimate the univariate analysis, along with logrank test in order to assess the differences of OS stratified by each variable. Cox proportional hazards model was used to conduct multivariate survival analysis. SPSS software (SPSS Inc., Chicago, USA, version 23) was used for statistical analysis, and GraphPad Prism 5 was used to generate the survival curve. A $P<0.05$ was considered as statistical significance.

\section{Results}

\section{Patient characteristics}

Of the 510607 patients with cancer in lung and bronchus from 2004-2013, $2972(0.58 \%)$ were diagnosed with LCNEC. In total, 1530 eligible patients were further enrolled in our research. The specific screening process was shown in Figure 1.To be specific, median age at diagnosis was 66 years (27-92years). Most of the patients were elderly people: 1105 patients $(72.2 \%)$ were $\geq 60$ years old, while 142 patients $(9.3 \%)$ were $\geq 80$ years old. There were more male patients than female (55.9\% VS. $44.1 \%)$. Only a small number of tumors $(4.2 \%, \mathrm{~N}=64)$ originated from the main bronchus, while most tumor occurred in upper lobe of the lung $(62.0 \%, \mathrm{~N}=948)$. Stage 
III-IV were noted in $65.5 \%$ of patients $(\mathrm{N}=1002)$, while $25.8 \%(\mathrm{~N}=395)$ and $8.7 \%(\mathrm{~N}=133)$ were stage I and II, respectively.

Based on the available information, $40.7 \%$ of patients $(\mathrm{N}=622)$ received cancer-directed surgery (CDS), including 133 patients receiving segmentectomy or wedge resection, 425 patients undergoing lobectomy or bilobectomy and 64 patients received pneumonectomy. In addition, 788(51.5\%) patients received chemotherapy and 565(36.9\%) patients were treated with radiotherapy.

\section{Overall survival and Prognostic factors}

The median follow-up time of all eligible patients was 11 months (range $0-131$ months).The 3-, 5-year OS and CSS rates were $22.8 \%, 16.8 \%$ and $26.5 \%, 20.8 \%$ respectively. And the OS and CSS curve was shown in figure 2 . In the univariate analyses, age $(P<0.001)$, sex $(P=0.001)$, primary $\operatorname{site}(P<0.001)$, differentiation $(P<0.001)$, AJCC stage groups 8 th edition $(P<0.001)$, surgery $(P<0.001)$, radiation $(P<0.001)$ were predictors of OS(Figure3). In addition, multivariate analysis further revealed that elderly $[(\geq 60$ and $<80$ years $) \mathrm{HR}: 1.203,95 \% \mathrm{CI}$ : 1.053 $1.375, P=0.007 ;(\geq 80$ years)HR:1.530, 95\%CI: $1.238-1.891, P<0.001]$, advanced AJCC stage [ (stage III)HR:2.606, 95\%CI: 2.083-3.260,P<0.001; (stage IV)HR:4.881，95\%CI: 3.923$6.072, P<0.001]$ were independent unfavorable prognostic factors, and that female (HR:0.845, 95\%CI: $0.754-0.947, P=0.004)$, surgery[(Segmentectomy/wedge resection) HR:0.526, 95\%CI: 0.413-0.669,P<0.001;(Lobectomy ／ Bilobectomy) HR:0.357, 95\%CI: $\quad 0.290-0.440$, $P<0.001$;(Pneumonectomy) HR:0.491, 95\%CI: 0.355-0.679,P<0.001], chemotherapy (HR:0.442, 95\%CI: $0.389-0.503, P<0.001$ ), and radiation (HR:0.837, 95\%CI: $0.738-0.949, P=0.005$ ) were 
independent favorable prognostic factors(Table 1).

\section{Discussion}

Lung neuroendocrine tumors are considered as a heterogeneous sub-type from pulmonary cancers, accounting for approximately $20 \%$ of all lung cancers, which include typical, atypical carcinoid, SCLC and LCNEC. The occurrence of LCNEC consists of $2.1 \%$ to $3.5 \%$ of all lung cancers [1]. Takei et al. reported similar rate being at 3.1\% (87/2790) [11]. These findings were in accordance with Iyoda et al. who reported LCNEC a rate of 3.4\% (72/2070) in resected lung cancers[12]. However, in our study, LCNEC accounted for only $0.58 \%$ of all lung cancers, which is a considerably lower than previous reports. The main reason for this discrepancy is that previous research data were only based on post-operative data from a single center; however, our study is a multicenter study with a wide range of coverage, including both patients with surgery and without surgery. Therefore, the proportion of LCNEC in all lung cancer might be even lower than previously thought.

Unlike other lung neuroendocrine tumors, LCNECs was often associated with male sex, older age, and cigarette consumption [13-15]. Furthermore, LCNEC was pathologically high-grade with poor-prognosis [16].These clinicopathological features are also found in our study. In addition, our study demonstrated that most patients with pulmonary LCNEC were at advanced stage when diagnosed.

Pulmonary LCNEC was biologically aggressive malignancies with a poor prognosis [17]. The 
5-year OS after resection of LCNEC had been reported to range from 13 to $57 \%[4,18,19]$. Prognosis was poor even in patients with potentially respectable stage I lung cancer, with 5-year survival rates ranging from $27 \%$ to $67 \%$ [17]. These previously-reported results are consistent with our findings.

Because of a low incidence, there are few reports concerning the prognostic factors of pulmonary LCNEC. Zhou et al. found that tumor location, resection status and EGFR mutational status were independent prognostic factors of LCNEC [6]. In our study, we found that elderly, male, and advanced stages are independent unfavorable prognostic factors and surgery, chemotherapy and radiation are protective factors for OS.

Primary surgery is the mainstream treatment for operable patients [1], which also facilitates in accurate diagnosis [20].Our research found surgery as a positive independent prognostic factor, which further confirmed the importance of surgery in pulmonary LCNEC. However, most patients with pulmonary LCNEC were not eligible for surgical resection due to systemic or local tumor metastasis. Only $40.7 \%$ of patients could receive CDS.

If surgery is not possible, radiotherapy and chemotherapy can be considered. The role of chemotherapy and radiotherapy in the treatment of local or advanced pulmonary LCNEC is still unclear [21]. Dresler et al[22] reported no survival benefits from postoperative chemotherapy, radiation therapy, or both in patients with resected LCNEC. Shimada et al. [23] found that the overall response rate to initial chemotherapy or chemoradiotherapy and the survival outcomes of high-grade neuroendocrine carcinoma — probable LCNEC were comparable to those of SCLC. In our study, we found that chemotherapy and radiotherapy were independently protective factors 
of pulmonary LCNEC.

Currently, the NCCN treatment guidelines suggest using the same approach as with other NSCLC tumors [24].Although, some researchers have found that treatment similar to SCLC was more appropriate than NSCLC for advanced LCNEC.[25]. Analysis of K-ras-2, p53, and C-raf-1 expressions has indicated pulmonary LCNEC are more genetically similar with SCLC, compared with NSCLC [26]. Furthermore, SCLC and pulmonary LCNEC are categorized as high grade neuroendocrine tumors, with similar clinical and histopathological characteristics. In addition, there were an increased number of scholars doubting if pulmonary LCNEC treatments being all similar to NSCLC was the right approach[27].

To our knowledge, our study is the largest retrospective analysis of LCNEC until now, with a total number of 1530 patients. This sample data are extracted from the SEER database, which is considered to be accurate and reliable. Through our research, physicians could have a better understanding of the clinicopathological features, survival and the treatment of patients with pulmonary LCNEC. However, there are certain limitations in our study. Although 11 variables were involved, there are still some important variables that SEER does not included, such as the chemotherapy regiments, blood tests, molecular test information and surgical margin status. Besides, selection bias might exist as we only included patients with complete information of involved variables.

\section{Conclusions}

In conclusion, our study finds that LCNEC has a higher incidence in elderly people, males, 
and advanced AJCC stage. In addition, elderly ( $\geq 60$ years), male, and advanced AJCC stages are independent unfavorable prognostic factors, while surgery chemotherapy and radiation are independent protective factors of OS.

\section{References}

[1] M. Fasano, C.M. Della Corte, F. Papaccio, F. Ciardiello, F. Morgillo, Pulmonary Large-Cell Neuroendocrine Carcinoma: From Epidemiology to Therapy, Journal of thoracic oncology : official publication of the International Association for the Study of Lung Cancer 10(8) (2015) 1133-41.

[2] W.D. Travis, R.I. Linnoila, M.G. Tsokos, C.L. Hitchcock, G.B. Cutler, Jr., L. Nieman, G. Chrousos, H. Pass, J. Doppman, Neuroendocrine tumors of the lung with proposed criteria for large-cell neuroendocrine carcinoma. An ultrastructural, immunohistochemical, and flow cytometric study of 35 cases, The American journal of surgical pathology 15(6) (1991) 529-53.

[3] N. Rekhtman, Neuroendocrine tumors of the lung: an update, Archives of pathology \& laboratory medicine 134(11) (2010) 1628-38.

[4] J.M. Varlotto, L.N. Medford-Davis, A. Recht, J.C. Flickinger, E. Schaefer, D.S. Zander, M.M. DeCamp, Should large cell neuroendocrine lung carcinoma be classified and treated as a small cell lung cancer or with other large cell carcinomas?, Journal of thoracic oncology : official publication of the International Association for the Study of Lung Cancer 6(6) (2011) 1050-8.

[5] C. Roesel, S. Terjung, G. Weinreich, T. Gauler, D. Theegarten, G. Stamatis, S. Welter, A Single-Institution Analysis of the Surgical Management of Pulmonary Large Cell Neuroendocrine Carcinomas, The Annals of thoracic surgery 101(5) (2016) 1909-14.

[6] F. Zhou, L. Hou, T. Ding, Q. Song, X. Chen, C. Su, W. Li, G. Gao, S. Ren, F. Wu, J. Fan, C. Wu, J. Zhang, C. Zhou, Distinct clinicopathologic features, genomic characteristics and survival of central and peripheral pulmonary large cell neuroendocrine carcinoma: From different origin cells?, Lung cancer (Amsterdam, Netherlands) 116 (2018) 30-37.

[7] M.A. Duggan, W.F. Anderson, S. Altekruse, L. Penberthy, M.E. Sherman, The Surveillance, Epidemiology, and End Results (SEER) Program and Pathology: Toward Strengthening the Critical Relationship, The American journal of surgical pathology 40(12) (2016) e94-e102.

[8] J. He, J. Shen, H. Pan, J. Huang, W. Liang, J. He, Pulmonary lymphoepithelioma-like carcinoma: a Surveillance, Epidemiology, and End Results database analysis, Journal of thoracic disease $7(12)(2015) 2330-8$.

[9] H.F. Sun, Y. Zhao, S.P. Gao, L.D. Li, W.Y. Fu, H.L. Jiang, M.T. Chen, L.P. Yang, W. Jin, Clinicopathological characteristics and survival outcomes of male breast cancer according to race: A SEER population-based study, Oncotarget 8(41) (2017) 69680-69690.

[10] J.R. Embry, M.G. Kelly, M.D. Post, M.A. Spillman, Large cell neuroendocrine carcinoma of the cervix: prognostic factors and survival advantage with platinum chemotherapy, Gynecologic oncology 120(3) (2011) 444-8.

[11] H. Takei, H. Asamura, A. Maeshima, K. Suzuki, H. Kondo, T. Niki, T. Yamada, R. Tsuchiya, Y. Matsuno, Large cell neuroendocrine carcinoma of the lung: a clinicopathologic study of eighty-seven cases, The Journal of thoracic and cardiovascular surgery 124(2) (2002) 285-92. 
[12] A. lyoda, K. Hiroshima, T. Toyozaki, Y. Haga, T. Fujisawa, H. Ohwada, Clinical characterization of pulmonary large cell neuroendocrine carcinoma and large cell carcinoma with neuroendocrine morphology, Cancer 91(11) (2001) 1992-2000.

[13] J. Sanchez de Cos Escuin, Diagnosis and treatment of neuroendocrine lung tumors, Archivos de bronconeumologia 50(9) (2014) 392-6.

[14] G. Selvaggi, G.V. Scagliotti, Histologic subtype in NSCLC: does it matter?, Oncology (Williston Park, N.Y.) 23(13) (2009) 1133-40.

[15] H. Asamura, T. Kameya, Y. Matsuno, M. Noguchi, H. Tada, Y. Ishikawa, T. Yokose, S.X. Jiang, T. Inoue, K. Nakagawa, K. Tajima, K. Nagai, Neuroendocrine neoplasms of the lung: a prognostic spectrum, Journal of clinical oncology : official journal of the American Society of Clinical Oncology 24(1) (2006) 70-6.

[16] Y. Oshiro, M. Kusumoto, Y. Matsuno, H. Asamura, R. Tsuchiya, H. Terasaki, H. Takei, A. Maeshima, S. Murayama, N. Moriyama, CT findings of surgically resected large cell neuroendocrine carcinoma of the lung in 38 patients, AJR. American journal of roentgenology 182(1) (2004) 87-91.

[17] A. Iyoda, K. Hiroshima, Y. Moriya, Y. Iwadate, Y. Takiguchi, T. Uno, Y. Nakatani, I. Yoshino, Postoperative recurrence and the role of adjuvant chemotherapy in patients with pulmonary large-cell neuroendocrine carcinoma, The Journal of thoracic and cardiovascular surgery 138(2) (2009) 446-53.

[18] A.B. Younossian, M.A. Brundler, M. Totsch, Feasibility of the new WHO classification of pulmonary neuroendocrine tumours, Swiss medical weekly 132(37-38) (2002) 53540 .

[19] R. Liang, T.X. Chen, Z.Q. Wang, K.W. Jin, L.Y. Zhang, Q.N. Yan, H.H. Zhang, W.P. Wang, A retrospective analysis of the clinicopathological characteristics of large cell carcinoma of the lung, Experimental and therapeutic medicine 9(1) (2015) 197-202.

[20] J. Zacharias, A.G. Nicholson, G.P. Ladas, P. Goldstraw, Large cell neuroendocrine carcinoma and large cell carcinomas with neuroendocrine morphology of the lung: prognosis after complete resection and systematic nodal dissection, The Annals of thoracic surgery 75(2) (2003) 348-52.

[21] K. Hiroshima, M. Mino-Kenudson, Update on large cell neuroendocrine carcinoma, Translational lung cancer research 6(5) (2017) 530-539.

[22] C.M. Dresler, J.H. Ritter, G.A. Patterson, E. Ross, M.S. Bailey, M.R. Wick, Clinical-pathologic analysis of 40 patients with large cell neuroendocrine carcinoma of the lung, The Annals of thoracic surgery 63(1) (1997) 180-5.

[23] Y. Shimada, S. Niho, G. Ishii, T. Hishida, J. Yoshida, M. Nishimura, K. Yoh, K. Goto, H. Ohmatsu, Y. Ohe, K. Nagai, Clinical features of unresectable high-grade lung neuroendocrine carcinoma diagnosed using biopsy specimens, Lung cancer (Amsterdam, Netherlands) 75(3) (2012) 368-73.

[24] D.E. Wood, E.A. Kazerooni, S.L. Baum, G.A. Eapen, D.S. Ettinger, L. Hou, D.M. Jackman, D. Klippenstein, R. Kumar, R.P. Lackner, L.E. Leard, I.T. Lennes, A.N.C. Leung, S.S. Makani, P.P. Massion, P. Mazzone, R.E. Merritt, B.F. Meyers, D.E. Midthun, S. Pipavath, C. Pratt, C. Reddy, M.E. Reid, A.J. Rotter, P.B. Sachs, M.B. Schabath, M.L. Schiebler, B.C. Tong, W.D. Travis, B. Wei, S.C. Yang, K.M. Gregory, M. Hughes, Lung Cancer Screening, Version 3.2018, NCCN Clinical Practice Guidelines in Oncology, Journal of the National Comprehensive Cancer Network : JNCCN 16(4) (2018) 412-441.

[25] J.M. Sun, M.J. Ahn, J.S. Ahn, S.W. Um, H. Kim, H.K. Kim, Y.S. Choi, J. Han, J. Kim, O.J. Kwon, Y.M. Shim, K. Park, Chemotherapy for pulmonary large cell neuroendocrine carcinoma: similar to that for small cell lung cancer or non-small cell lung cancer?, Lung cancer (Amsterdam, Netherlands) 77(2) (2012) 365-70.

[26] R.M. Przygodzki, S.D. Finkelstein, J.C. Langer, P.A. Swalsky, N. Fishback, A. Bakker, D.G. Guinee, M. Koss, W.D. Travis, Analysis of p53, K-ras-2, and C-raf-1 in pulmonary neuroendocrine tumors. Correlation with histological subtype and clinical outcome, The American journal of pathology 148(5) (1996) $1531-41$.

[27] F.G. Fernandez, R.J. Battafarano, Large-cell neuroendocrine carcinoma of the lung: an aggressive neuroendocrine lung cancer, Seminars in thoracic and cardiovascular surgery $18(3)(2006) 206-10$. 


\section{Figure Legends}

325

326

327

328

329

Figure 1.Flow chart for screening eligible patients.

Figure2. Kaplan-Meier survival plots for eligible patients showing A:Overall survival (OS) and B:Disease-specific survival (DSS)

Figure3.Kaplan-Meier survival curves of some covariates without adjusted. A: age at diagnosis; B:Sex; C:8th AJCC stage; D:surgery ; E:chemotherapy; F:radiation. 


\section{Figure 1}

Figure1

Figure1.Flow chart for screening eligible patients.

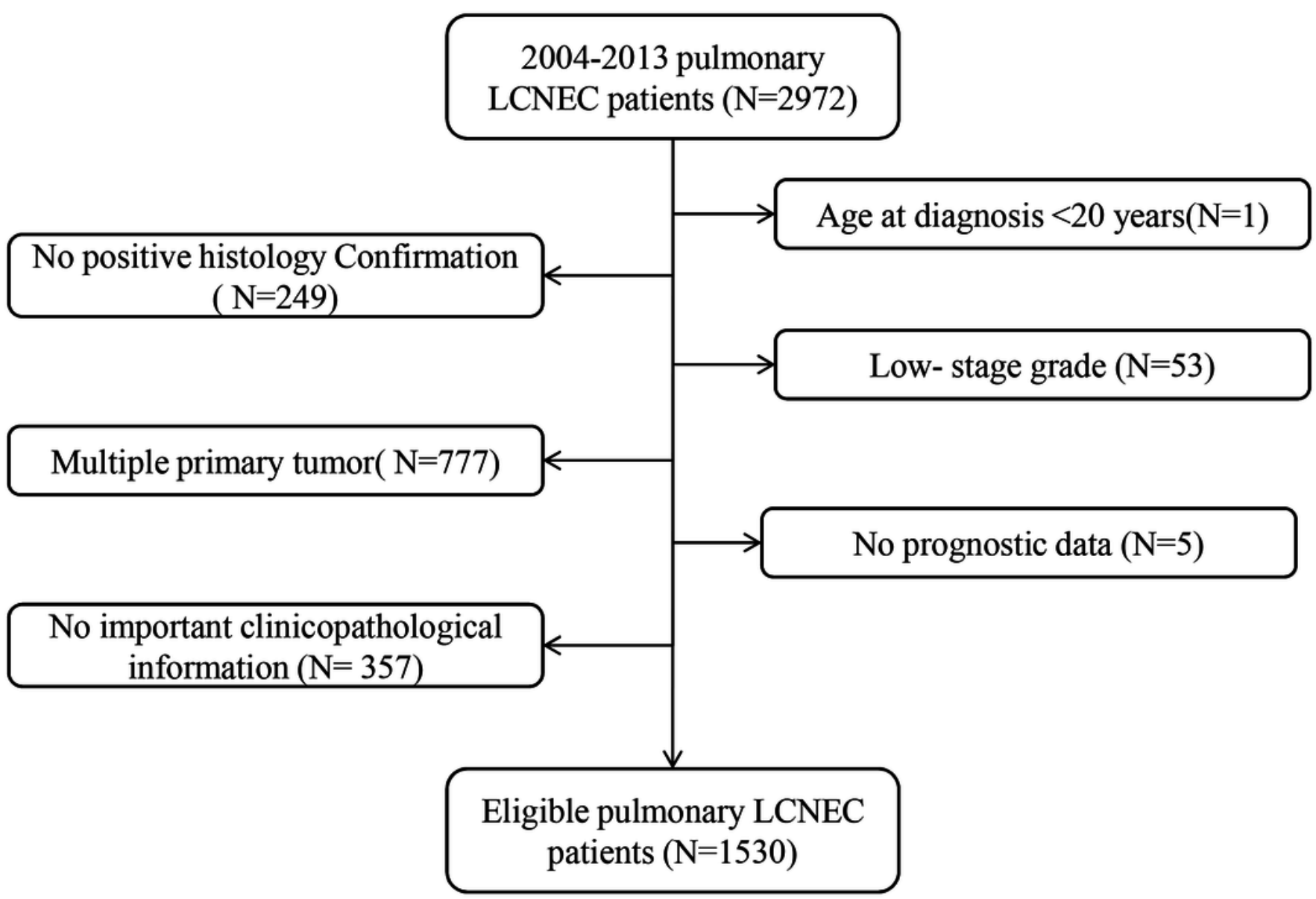


Figure 2

Figure2

Figure2. Kaplan-Meier survival plots for eligible patients showing A: Overall survival (OS) and

B: Disease-specific survival (DSS)
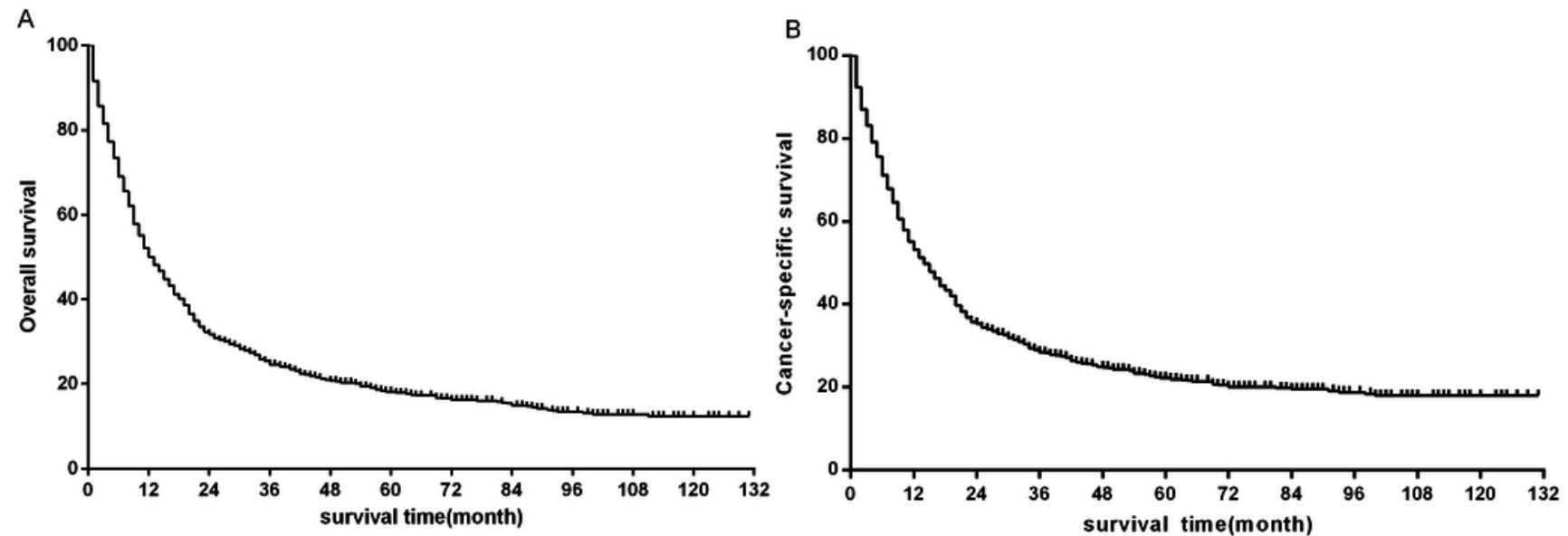
Figure 3

Figure3

Figure3.Kaplan-Meier survival curves of some covariates without adjusted. A:age at diagnosis; B:Sex; C:summary stage; D:surgery ; E:chemotherapy; F:radiation.
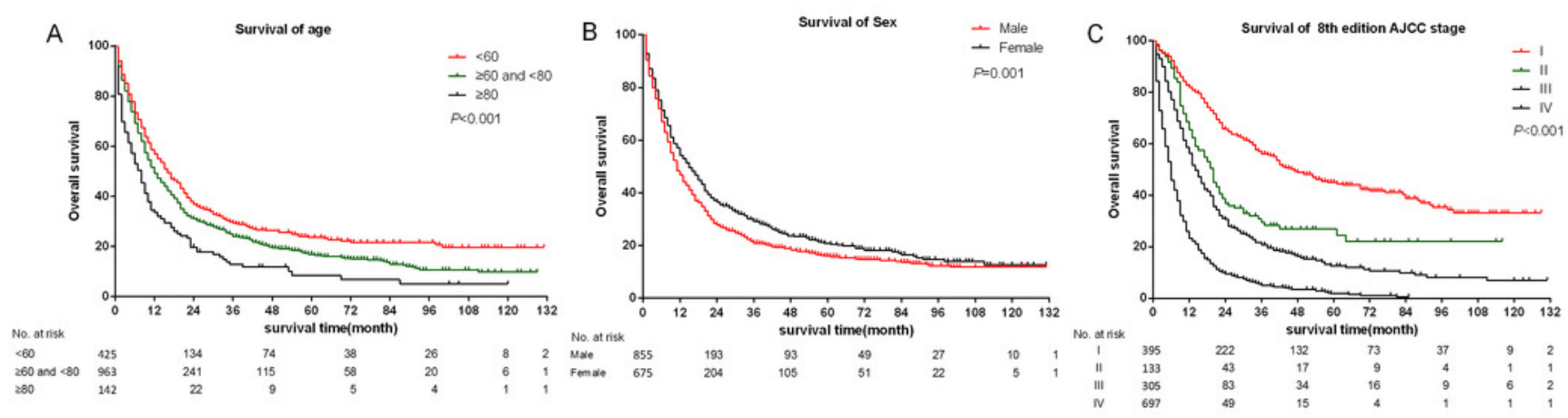

D

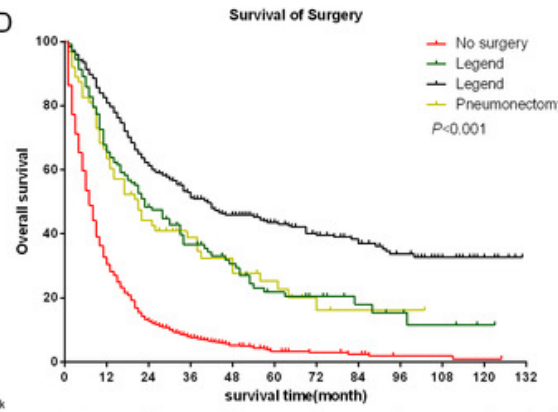

E
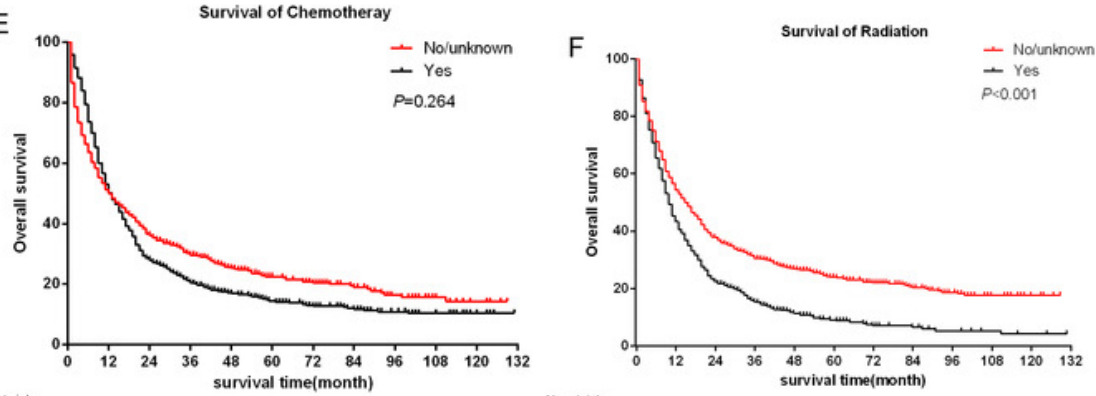

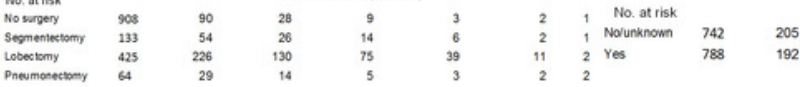




\section{Table $\mathbf{1}$ (on next page)}

Table 1

Table 1 Univariate and multivariate analyses of overall survival (OS) in the eligible patients. 
1 Table 1 Univariate and multivariate analyses of overall survival (OS) in the eligible patients.

\begin{tabular}{|c|c|c|c|c|}
\hline \multirow{2}{*}{ Variables } & \multirow{2}{*}{$\mathrm{N}(\%)$} & \multirow{2}{*}{$\begin{array}{c}\text { Univariate analysis } \\
P \text {-value }\end{array}$} & \multicolumn{2}{|c|}{ Multivariate analysis } \\
\hline & & & $\mathrm{HR}(95 \% \mathrm{CI})$ & $P$-value \\
\hline Age & & $<0.001$ & & $<0.001$ \\
\hline$<60$ & $425(27.8 \%)$ & & Reference & \\
\hline$\geq 60$ and $<80$ & $963(62.9 \%)$ & & $1.203(1.053,1.375)$ & 0.007 \\
\hline$\geq 80$ & $142(9.3 \%)$ & & $1.530(1.238,1.891)$ & $<0.001$ \\
\hline Sex & & 0.001 & & 0.004 \\
\hline Male & $855(55.9 \%)$ & & Reference & \\
\hline Female & $675(44.1 \%)$ & & $0.845(0.754,0.947)$ & \\
\hline Race & & 0.885 & & NI \\
\hline White & $1283(83.9 \%)$ & & & \\
\hline Black & $187(12.2 \%)$ & & & \\
\hline Other ${ }^{\#}$ & $60(3.9 \%)$ & & & \\
\hline Marital status & & 0.276 & & NI \\
\hline Married & $839(54.8 \%)$ & & & \\
\hline Unmarried & $691(45.2 \%)$ & & & \\
\hline Laterality & & 0.289 & & NI \\
\hline Bilateral & $4(0.4 \%)$ & & & \\
\hline Left & $623(40.3 \%)$ & & & \\
\hline Right & $903(59.3 \%)$ & & & \\
\hline Primary site & & $<0.001$ & & 0.443 \\
\hline Main bronchus & $64(4.2 \%)$ & & Reference & \\
\hline Upper lobe & $948(62.0 \%)$ & & $0.917(0.700,1.202)$ & 0.531 \\
\hline Middle lobe & $82(5.4 \%)$ & & $1.118(0.793,1.577)$ & 0.524 \\
\hline Lower lobe & $408(26.7 \%)$ & & $0.891(0.673,1.181)$ & 0.423 \\
\hline Overlapping lesion & $28(1.8 \%)$ & & $0,836(0.507,1.377)$ & 0.482 \\
\hline Differentiation & & $<0.001$ & & 0.322 \\
\hline Grade III & $586(38.3 \%)$ & & Reference & \\
\hline Grade IV & $191(12.5 \%)$ & & $1.157(0.957,1.399)$ & 0.133 \\
\hline Unknown & $753(49.2 \%)$ & & $1.035(0.909,1.179)$ & 0.603 \\
\hline AJCC stage groups 8th edition & & $<0.001$ & & $<0.001$ \\
\hline I & $395(25.8 \%)$ & & Reference & \\
\hline II & $133(8.7 \%)$ & & $2.619(2.027,3.384)$ & $<0.001$ \\
\hline III & $305(19.9 \%)$ & & $2.606(2.083,3.260)$ & $<0.001$ \\
\hline IV & $697(45.6 \%)$ & & $4.881(3.923,6.072)$ & $<0.001$ \\
\hline Surgery & & $<0.001$ & & $<0.001$ \\
\hline No surgery & $908(59.3 \%)$ & & Reference & \\
\hline Segmentectomy/wedge resection & $133(8.7 \%)$ & & $0.526(0.413,0.669)$ & $<0.001$ \\
\hline Lobectomy / Bilobectomy & $425(27.8 \%)$ & & $0.357(0.290,0.440)$ & $<0.001$ \\
\hline Pneumonectomy & $64(4.2 \%)$ & & $0.491(0.355,0.679)$ & $<0.001$ \\
\hline
\end{tabular}


Chemotheray

No/unknown

Yes

Radiation

No/unknown

Yes
0.264

$742(48.5 \%)$

$788(51.5 \%)$

965(63.1\%)

$565(36.9 \%)$

$<0.001$
$<0.001$

Reference

$0.442(0.389,0.503)$

Reference

$0.837(0.738,0.949)$

2 \#Other: American Indian/AK Native, Asian/Pacific Islander. Abbreviations: N: number; HR, hazard ratio; 95\% CI, 95\% 3 confidence index; NI: not included in the multivariate survival analysis. 